\title{
Patient-Reported Outcomes in the Phase III BRIGHTE Trial of the HIV-1 Attachment Inhibitor Prodrug Fostemsavir in Heavily Treatment-Experienced Individuals
}

\author{
Sarah-Jane Anderson ${ }^{1,2}$ (1) Miranda Murray ${ }^{2} \cdot$ David Cella $^{3} \cdot$ Robert Grossberg $^{4} \cdot$ Debbie Hagins $^{5} \cdot$ William Towner $^{6}$. \\ Marcia Wang ${ }^{7} \cdot$ Andrew Clark $^{2} \cdot$ Amy Pierce $^{8} \cdot$ Cyril Llamoso $^{9} \cdot$ Peter Ackerman $^{9} \cdot$ Max Lataillade $^{9}$
}

Accepted: 7 June 2021 / Published online: 28 June 2021

(C) The Author(s) 2021

\begin{abstract}
Introduction Heavily treatment-experienced (HTE) people living with HIV-1 (PLWH) have limited viable antiretroviral regimens available because of multidrug resistance and safety concerns. The first-in-class HIV-1 attachment inhibitor fostemsavir demonstrated efficacy and safety in HTE participants in the ongoing phase III BRIGHTE trial.

Objectives We describe patient-reported outcomes (PROs) through week 48.

Methods Eligible participants for whom their current regimen was failing were assigned to the randomized cohort (RC; one to two fully active agents remaining) or the nonrandomized cohort (NRC; no fully active agents remaining). PRO assessments included the EQ-5D-3L, EQ-VAS, and Functional Assessment of HIV Infection (FAHI) instruments.

Results Both cohorts achieved increases in EQ-5D-3L US- and UK-referenced utility score from baseline at week 24. Mean visual analog scale (VAS) scores in the RC and NRC increased from baseline by 8.7 (95\% CI 6.2-11.2) and 5.6 points (95\% CI 1.5-9.7) at week 24 and increased from baseline by 9.8 (95\% CI 7.0-12.6) and 4.9 points (95\% CI 0.6-9.2) at week 48, respectively. Mean increases in FAHI total score from baseline to weeks 24 and 48 in the RC were 6.9 (95\% CI 4.2-9.7) and 5.8 (95\% CI 2.7-9.0), respectively, whereas mean increases in physical and emotional well-being subscale scores were 2.7 (95\% CI 1.9-3.6) and 2.4 (95\% CI 1.3-3.4) and 3.2 (95\% CI 2.2-4.2) and 2.6 (95\% CI 1.6-3.7), respectively, with little to no change in other subscales.

Conclusions Improvements in major domains of the EQ-VAS and FAHI through week 48, combined with efficacy and safety results, support the use of fostemsavir for HTE PLWH.
\end{abstract}

Trial Registration Number and Date NCT02362503; February 13, 2015.

Sarah-Jane Anderson

sarah-jane.x.anderson@gsk.com

1 GlaxoSmithKline, Brentford, UK

2 ViiV Healthcare, 980 Great West Road, Brentford, Middlesex TW8 9GS, UK

3 Northwestern University Feinberg School of Medicine, Chicago, IL, USA

4 Montefiore Medical Center, Bronx, NY, USA

5 Georgia Department of Public Health, Coastal Health District, Chatham CARE Center, Savannah, GA, USA

6 Southern California Kaiser Permanente Medical Group, Los Angeles, CA, USA

7 GlaxoSmithKline, Upper Providence, PA, USA

8 ViiV Healthcare, Research Triangle Park, NC, USA

9 ViiV Healthcare, Branford, CT, USA 


\section{Key Points For Decision Makers}

Patient-reported outcomes can show treatment benefits by measuring adherence to therapy, health status, and health-related quality of life (HRQOL) in people with HIV-1 infection.

A heavily treatment-experienced (HTE) population with HIV-1 may have reduced HRQOL because of challenges achieving virologic suppression, reduced immune function, overlapping toxicities, and drug-drug interactions, which can occur with many lines of therapy.

Results from the BRIGHTE study of fostemsavir showed improvements in HRQOL in a diverse population of HTE individuals with HIV-1, many of whom have extensive antiretroviral therapy drug resistance and limited treatment options.

\section{Introduction}

Antiretroviral therapy (ART) has greatly reduced morbidity and mortality rates in people living with HIV (PLWH) [1]. However, a number of heavily treatment-experienced (HTE) individuals have limited treatment options remaining because of multidrug resistance, prior intolerance, contraindications, or other safety concerns. Thus, there is a need to develop effective and well-tolerated therapies without crossresistance to currently available therapies to address ongoing treatment challenges for HTE PLWH.

Fostemsavir is a first-in-class attachment inhibitor prodrug that is metabolized to its active moiety, temsavir [2]. Temsavir binds directly to the HIV-1 viral envelope glycoprotein 120 , locking it in a closed conformational state that inhibits initial attachment to cell-surface CD4+ receptors, thereby preventing entry into and infection of host immune cells [3]. Fostemsavir has no in vitro cross-resistance to other ART agents and is active regardless of HIV-1 tropism, including $\mathrm{C}-\mathrm{C}$ chemokine receptor type 5 , C-X-C chemokine receptor type 4, and dual-tropic HIV-1 strains [4-6].

Fostemsavir was approved by the US FDA in July 2020 for the treatment of HTE adults with multidrug-resistant HIV-1 infection for whom their current antiretroviral regimen was failing [7] on the basis of efficacy and safety results from the ongoing phase III BRIGHTE study, which is being conducted in HTE PLWH. In the BRIGHTE study, HTE participants in the randomized cohort (RC) had one to no more than two fully active ART classes available, and participants in the nonrandomized cohort (NRC) had no fully active ART classes available. Participants in the NRC were allowed to enroll in other trials of antiretroviral drugs, allowing for the potential inclusion of additional investigational antiretroviral drugs in their optimized background therapy (OBT) [8]. In the primary endpoint (HIV-1 RNA $\log _{10}$ change at day 8), fostemsavir showed superior efficacy relative to placebo in decreasing median HIV-1 RNA in the RC at day 8 (fostemsavir $-0.79 \log _{10}$ copies/ $\mathrm{mL}$; placebo $-0.17 \log _{10}$ copies $/ \mathrm{mL}$; treatment difference -0.63 [95\% confidence interval $\{\mathrm{CI}\}-0.81$ to -0.44 ]; $P<0.001$ ) [8]. At week $24,53 \%$ of RC participants and $37 \%$ of NRC participants achieved virologic suppression (HIV-1 RNA $<40$ copies/mL by the FDA snapshot algorithm). Efficacy was maintained through week 48, with $54 \%$ of participants in the RC and $38 \%$ in the NRC achieving virologic suppression. HIV-1 RNA $<200$ copies $/ \mathrm{mL}$ at 24 and 48 weeks was achieved by 79 and $84 \%$ of participants in the RC and 53 and 59\% in the NRC, respectively. CD4+ T cell count generally increased over time in both cohorts. By week 48, mean CD4+ T-cell count increased from baseline by 138.9 and 63.5 cells $/ \mathrm{mm}^{3}$ in the $\mathrm{RC}$ and NRC cohorts, respectively. Fostemsavir was well-tolerated with few discontinuations due to adverse events (AEs). The most common AEs overall were diarrhea $(n=83$ [22\%]), nausea $(n=60[16 \%])$, upper respiratory tract infection $(n=50[13 \%])$, headache $(n=46[12 \%])$, cough $(n=43[12 \%])$, and nasopharyngitis $(n=43[12 \%])$.

In addition to efficacy and safety, patient-reported outcome (PRO) measures are important endpoints of a clinical trial that can demonstrate a direct treatment benefit by measuring an individual's adherence to therapy, health status, and health-related quality of life (HRQOL) [9, 10]. These concepts are important to measure in an HTE population who may have been taking ART for many years and could have experienced numerous treatment changes that can affect treatment success, lead to the emergence of drug resistance, and reduce available treatment options $[11,12]$. Consequently, there may be greater challenges in achieving virologic suppression in this population, and these individuals commonly have reduced immune function (reflected by low CD4 + T-cell counts); elevated viral load and reduced immune function have a negative impact on HRQOL [13-16]. Furthermore, compared with PLWH on first- or second-line therapy, HTE PLWH may have reduced HRQOL because of overlapping toxicities and drug-drug interactions common with later lines of therapy $[17,18]$. Given the observed clinical improvements in BRIGHTE trial participants and that fostemsavir was generally well-tolerated, these analyses seek to explore the impact of fostemsavir treatment on HRQOL [8]. Herein, we describe PROs, including general and disease-specific HRQOL assessments, through the week 48 analysis of the BRIGHTE study. 


\section{Methods}

\subsection{Study Design and Study Participants}

The BRIGHTE study (ClinicalTrials.gov identifier NCT02362503) is a two-cohort, phase III trial conducted across 108 investigational sites in 23 countries. Enrollment occurred between February 2015 and May 2016. Detailed methods and trial design have previously been published [8]. Briefly, eligible participants were HTE adult PLWH (aged $\geq 18$ years) with no more than two antiretroviral classes remaining and failure of the current regimen (confirmed HIV-1 RNA $\geq 400$ copies/mL) [8]. Participants were assigned to the $\mathrm{RC}$ if one to no more than two fully active ART classes were available or to the NRC if no fully active ART classes were available. An ART class was considered available if one or more agents within that class were fully active and acceptable (per prior intolerance, contraindication, and other safety concerns). Participants in the $\mathrm{RC}$ were randomized 3:1 to begin a period of functional monotherapy in which they received blinded fostemsavir $600 \mathrm{mg}$ or blinded placebo twice daily with their current failing regimen from days 1 to 8 . After 8 days, all participants in the $\mathrm{RC}$ received open-label fostemsavir $600 \mathrm{mg}$ twice daily in combination with an OBT chosen at the discretion of the managing investigator. In the NRC, participants received open-label fostemsavir $600 \mathrm{mg}$ twice daily in combination with OBT throughout the study. Participants in the NRC were permitted to coenroll in other investigational antiretroviral trials, but those in the RC were not. Although changes in OBT did not mandate discontinuation from the study, standard snapshot analysis rules applied (e.g., any alteration in background therapy because of lack of efficacy was considered virologic failure for both cohorts). The primary endpoint was the mean change in $\log _{10}$ HIV-1 RNA from baseline to day 8 in the RC. Participants remained in the trial despite meeting protocol-defined virologic failure criteria, and those who requested to discontinue study drug were instructed to remain in the study for follow-up procedures, including PRO survey administration. As such, a range of clinical outcomes are represented across study participants.

\subsection{Patient-Reported Outcome Assessments}

HRQOL was assessed based on prior research linking CD4 + T-cell count and viral load with HRQOL in PLWH $[14,15]$. Assessments of PROs included a generic adherence questionnaire, the Modified-Medication Adherence Self-Report Inventory (M-MASRI) [19], the EQ-5D-3L [20], and the Functional Assessment of HIV Infection
(FAHI), an HRQOL questionnaire specific to PLWH [16]. The EQ-5D-3L was selected based on its use for measuring HRQOL in the general population and in PLWH [21]. The FAHI was selected based on its validity and previous use in PLWH, including HTE PLWH $[15,16,22]$. The M-MASRI is a self-reported questionnaire used to determine the percentage of days of medication taken and the percentage of doses taken within $2 \mathrm{~h}$ of the correct time. The EQ-5D-3L covers five dimensions of mobility, pain, self-care, usual activities, and anxiety/depression, with three levels of response for each dimension (no, some, or extreme problems). The FAHI assesses physical wellbeing, functional and global well-being, emotional wellbeing/living with HIV, social well-being, and cognitive functioning [22].

The PRO assessments were administered on paper, in the clinic. The M-MASRI questionnaire was administered at baseline and week 24. The EQ-5D-3L and FAHI instruments were administered at baseline and weeks 12, 24, 36, and 48, and every 12 weeks thereafter. PRO data were combined for both subgroups in the RC because a difference in PROs between the groups was not expected to be seen from week 12 onward because, after day 8 , all participants received fostemsavir $600 \mathrm{mg}$ twice daily in combination with OBT.

The M-MASRI was modified for the RC at day 8 to ask about the previous 7 days rather than 1 month. Each component is summarized as proportions in the following categories: $<50 \%, 50$ to $<60 \%, 60$ to $<70 \%, 70$ to $<80 \%, 80$ to $<90 \%, 90$ to $<95 \%$, and $\geq 95 \%$.

The EQ-5D-3L includes five health status questions and an associated visual analog scale (EQ-VAS) [20]. The response results in a one-digit number expressing the level selected for that dimension. The numbers for the five dimensions can be combined into a five-digit number that represents the state of the respondent's health. The scores can then be converted to a summary index utility score. The EQVAS is a self-rated assessment of overall health on a vertical VAS with endpoints of 0 (worst imaginable health state) to 100 (best imaginable health state). Respondents mark an " $\mathrm{X}$ " on the scale to indicate their health status, and the corresponding number on the scale is their score.

The FAHI is a 47-item questionnaire grouped into five subscales assessing well-being [22]. Each item is scored on a scale of $0-4$; subscale and total scores can be derived, with the total score ranging from 0 to 176 . Higher scores are associated with better HRQOL.

\subsection{Statistical Analysis}

The EQ-5D-3L endpoints included two utility scores derived from US and UK value sets. Both the US and UK value sets are based on transformed time trade-offbased preferences for 42 EQ-5D health states; however, 
they are based on respondents from the US and UK general populations, respectively [24]. Although the same 42 health states are used in the US and UK value sets, the transformation method used differs and leads to different preference values. The transformative method used in the US value set is more similar to expected utility theory and results in prediction of higher EQ-5D health states than the UK value set. The FAHI endpoints included a total score and individual subscale scores (for which four is always the best [healthiest] possible score) expressed as the sum of the 0-4 response ratings. Values and changes from baseline were summarized using descriptive statistics. No statistical testing was performed as part of the prespecified analyses. Post hoc analyses were conducted to determine $95 \%$ CIs. In addition, post hoc analyses were conducted to investigate the change in PRO measures in various subgroups of participants. Subgroups were defined based on participant clinical and demographic characteristics, and the RC and NRC were pooled for subgroup analyses.

\subsection{Ethics}

The study is being performed in accordance with good clinical practice, as defined in the International Conference on Harmonization and in accordance with the ethical principles underlying EU Directive 2001/20/EC and the US Code of Federal Regulations, Title 21, Part 50. Each site conducted the study according to the protocol with oversight from an institutional review board or independent ethics committee. All participants provided informed consent in agreement with the Declaration of Helsinki principles.

\section{Results}

\subsection{Participant Demographics and Study Disposition}

Of the 731 participants who were screened, 371 were enrolled and treated with at least one dose of fostemsavir; 272 were enrolled in the RC (69 placebo, 203 fostemsavir) and 99 in the NRC (Fig. 1). The two most common reasons for screening failure were having more than two

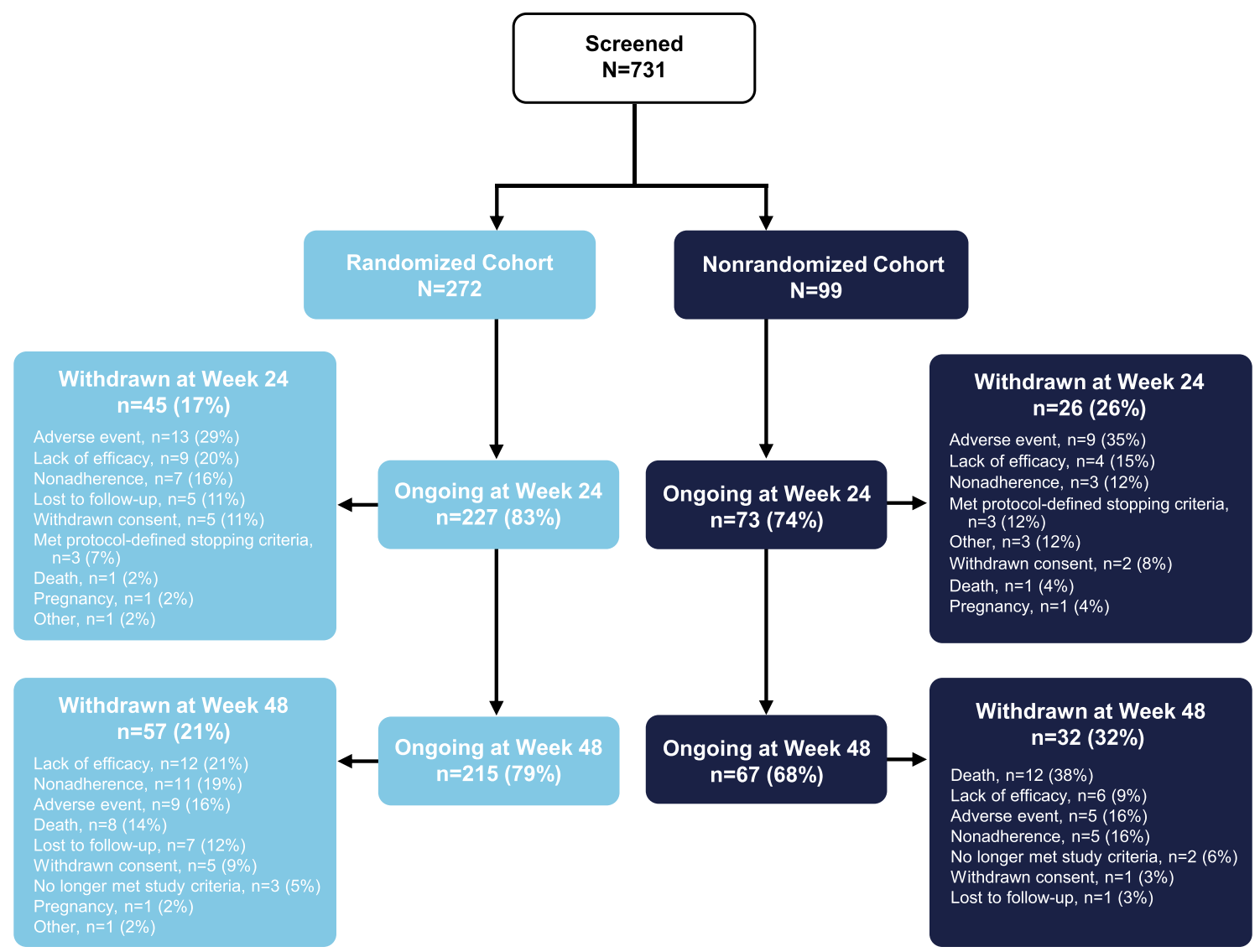

Fig. 1 BRIGHTE study disposition at week 24 and week 48 
Table 1 Participant demographics

\begin{tabular}{|c|c|c|c|c|}
\hline \multirow[t]{2}{*}{ Parameter } & \multicolumn{2}{|l|}{ Randomized cohort } & \multirow{2}{*}{$\begin{array}{l}\text { Nonrandomized cohort } \\
\text { FTR } 600 \mathrm{mg} \text { BID }(n=99)\end{array}$} & \multirow{2}{*}{$\begin{array}{l}\text { Total } \\
\text { Total }(N=371)\end{array}$} \\
\hline & Placebo BID $(n=69)$ & $\begin{array}{l}\text { FTR } 600 \mathrm{mg} \text { BID } \\
(n=203)\end{array}$ & & \\
\hline Age, years & $45(19-66)$ & $48(18-73)$ & $50(17-72)$ & $49(17-73)$ \\
\hline$<50$ & $46(67)$ & $116(57)$ & $44(44)$ & $206(56)$ \\
\hline \multicolumn{5}{|l|}{ Sex } \\
\hline Male & $57(83)$ & $143(70)$ & $89(90)$ & $289(78)$ \\
\hline \multicolumn{5}{|l|}{ Race } \\
\hline White & $47(68)$ & $137(67)$ & $73(74)$ & $257(69)$ \\
\hline Black/African American & $18(26)$ & $42(21)$ & $23(23)$ & $83(22)$ \\
\hline American Indian or Alaska Native & $1(1)$ & $6(3)$ & $1(1)$ & $8(2)$ \\
\hline Asian & 0 & $2(<1)$ & 0 & $2(<1)$ \\
\hline Native Hawaiian or other Pacific Islander & 0 & $1(<1)$ & 0 & $1(<1)$ \\
\hline Other & $3(4)$ & $15(7)$ & $2(2)$ & $20(5)$ \\
\hline Baseline HIV-1 RNA, $\log _{10}$ copies/mL & $4.5(1.6-6.9)$ & $4.7(1.6-6.4)$ & $4.3(1.6-6.6)$ & $4.6(1.6-6.9)$ \\
\hline \multicolumn{5}{|l|}{ Baseline HIV-1 RNA, copies/mL } \\
\hline$<400$ & $7(10)$ & $14(7)$ & $5(5)$ & $26(7)$ \\
\hline 400 to $<1000$ & $3(4)$ & $7(3)$ & $4(4)$ & $14(4)$ \\
\hline 1000 to $<100,000$ & $35(51)$ & $126(62)$ & $75(76)$ & $236(64)$ \\
\hline$\geq 100,000$ & $24(35)$ & $56(28)$ & $15(15)$ & $95(26)$ \\
\hline Baseline CD4 + T-cell count, cells $/ \mathrm{mm}^{3}$ & $100(0-915)$ & $99(0-1160)$ & $41(0-641)$ & $80(0-1160)$ \\
\hline \multicolumn{5}{|l|}{ Baseline CD4 + T-cell count, cells $/ \mathrm{mm}^{3}$} \\
\hline$<20$ & $17(25)$ & $55(27)$ & $40(40)$ & $112(30)$ \\
\hline 20 to $<50$ & $6(9)$ & $19(9)$ & $14(14)$ & $39(11)$ \\
\hline 50 to $<200$ & $26(38)$ & $76(37)$ & $25(25)$ & $127(34)$ \\
\hline 200 to $<500$ & $16(23)$ & $42(21)$ & $18(18)$ & $76(20)$ \\
\hline$\geq 500$ & $4(6)$ & $11(5)$ & $2(2)$ & $17(5)$ \\
\hline \multicolumn{5}{|l|}{ Years treated for HIV-1 infection } \\
\hline$\leq 10$ & $14(20)$ & $27(13)$ & $5(5)$ & $46(12)$ \\
\hline $11-15$ & $14(20)$ & $30(15)$ & $11(11)$ & $55(15)$ \\
\hline $16-20$ & $18(26)$ & $72(35)$ & $22(22)$ & $112(30)$ \\
\hline$>20$ & $22(32)$ & $70(34)$ & $58(59)$ & $150(40)$ \\
\hline Unknown & $1(1)$ & $4(2)$ & $3(3)$ & $8(2)$ \\
\hline AIDS history & $46(67)$ & $131(65)$ & $71(72)$ & $248(67)$ \\
\hline
\end{tabular}

Data are presented as $\mathrm{n}(\%)$ or median (range) unless otherwise indicated

$B I D$ twice daily, FTR fostemsavir

antiretroviral classes remaining or the current ART regimen not failing (lack of confirmed plasma HIV-1 RNA $\geq 400$ copies $/ \mathrm{mL}$ ). Baseline demographic characteristics were similar across both cohorts (Table 1) [8]. The median age of participants was 49 years, $78 \%$ were male, and $69 \%$ were white. Of the total study participants, $71 \%$ had been treated for HIV-1 infection for $>15$ years, and $67 \%$ had a prior history of AIDS. Relative to the RC, a greater proportion of participants in the NRC had baseline CD4 + T-cell counts $<20$ cells $/ \mathrm{mm}^{3}$ (NRC $40 \%$; RC 26\%) and had been treated for HIV-1 for $>20$ years (NRC 59\%; RC 34\%).

In general, participants in both cohorts had extensive treatment history with several antiretroviral classes
(Table 2). Specifically, most participants had treatment history with nucleoside reverse transcriptase inhibitors (RC 99\%; NRC 100\%), protease inhibitors (RC 94\%; NRC 99\%), and non-nucleoside reverse transcriptase inhibitors (RC 92\%; NRC 96\%). Relative to the RC, a greater proportion of participants in the NRC had experience with integrase inhibitors (RC 75\%; NRC 95\%), entry inhibitors (RC $39 \%$; NRC 69\%), and CCR5 antagonists (RC 26\%; NRC $40 \%$ ). The most common OBT component at the beginning of the open-label phase in the RC was dolutegravir $(n=229$ [84\%]). The most common OBT components in the NRC were dolutegravir $(n=74[75 \%])$, tenofovir $(n=74[75 \%])$, and darunavir $(n=71[72 \%])$. 
Table 2 Antiretroviral therapy class history

\begin{tabular}{lllll}
\hline Medication & \multicolumn{2}{l}{ Randomized cohort } & Nonrandomized cohort & Total \\
\cline { 2 - 3 } & Placebo $(n=69)$ & $\begin{array}{l}\text { FTR 600 mg } \\
\text { BID }(n=203)\end{array}$ & FTR 600 mg BID $(n=99)$ & Total $(N=371)$ \\
\hline Any medication & $69(100)$ & $203(100)$ & $99(100)$ & $371(100)$ \\
INI & $54(78)$ & $149(73)$ & $94(95)$ & $297(80)$ \\
NRTI & $67(97)$ & $202(>99)$ & $99(100)$ & $368(>99)$ \\
PI $^{\mathrm{a}}$ & $64(93)$ & $193(95)$ & $98(99)$ & $355(96)$ \\
NNRTI & $61(88)$ & $189(93)$ & $95(96)$ & $345(93)$ \\
Entry inhibitor & $26(38)$ & $81(40)$ & $68(69)$ & $175(47)$ \\
CCR5 antagonist & $20(29)$ & $52(26)$ & $40(40)$ & $112(30)$ \\
Other ART & $7(10)$ & $24(12)$ & $21(21)$ & $52(14)$ \\
\hline
\end{tabular}

Data are presented as $\mathrm{n}(\%)$

$A R T$ antiretroviral therapy, BID twice daily, FTR fostemsavir, INI integrase inhibitor, NNRTI non-nucleoside reverse transcriptase inhibitor, NRTI nucleoside reverse transcriptase inhibitor, $P I$ protease inhibitor

${ }^{\mathrm{a}}$ With or without a boosting agent
Of the 272 participants in the RC, 45 (17\%) had discontinued by week 24 ; of the 99 participants in the NRC, 26 (26\%) had discontinued by week 24 . By the week 48 data lock, 57 (21\%) and $32(32 \%)$ participants had discontinued in the RC and NRC, respectively. The most common reasons for discontinuation were lack of efficacy (RC 21\%; NRC 19\%), nonadherence (RC 19\%; NRC 16\%), AEs (RC $16 \%$; NRC 16\%), and death (RC 14\%; NRC 38\%).

\subsection{Efficacy and Safety}

Efficacy and safety analyses demonstrated the superior efficacy of fostemsavir (added to failing regimen) compared with placebo through 8 days of functional monotherapy [8]. At weeks 24 and 48, virologic success (<40 copies/ $\mathrm{mL}$, intention-to-treat snapshot analysis) was observed in 53 and $54 \%$ of the RC and 37 and $38 \%$ of the NRC, respectively. Fostemsavir was generally well-tolerated, with low rates of AEs leading to discontinuation.

\subsection{Modified-Medication Adherence Self-Report Inventory}

In the month before baseline, participants in the RC $(n=255)$ and NRC $(n=93)$ took their ART medication for an average of 88 and $93 \%$ of days, respectively, with the majority of doses (84\% [ $n=254]$ and $85 \%$ [ $n=93]$, respectively) taken within $2 \mathrm{~h}$ of the correct time. At week 24, participants in the RC $(n=225)$ and NRC $(n=84)$ took their ART medication for an average of 96 and $95 \%$ of days, respectively, during the last month; 91\% $(n=225)$ and $89 \%(n=84)$ of doses, respectively, were taken within $2 \mathrm{~h}$ of the correct time (Table 3).

\subsection{EQ-5D-3L and EQ-VAS}

The EQ-5D-3L baseline mean \pm standard deviation (SD) utility scores were nominally higher in the RC (US value set $0.83 \pm 0.17$; UK value set $0.78 \pm 0.24$ ) than in the NRC (US value set $0.80 \pm 0.20$; UK value set $0.74 \pm 0.28$ ); a similar pattern was observed for the EQ-VAS (RC $74.7 \pm 21.6$;

Table 3 Results by cohort for modified-medication adherence self-report inventory (ITT-E population)

\begin{tabular}{|c|c|c|c|c|c|c|}
\hline \multirow[t]{2}{*}{ Parameter } & \multicolumn{2}{|c|}{$\begin{array}{l}\text { Randomized cohort } \\
(n=272)\end{array}$} & \multicolumn{2}{|c|}{$\begin{array}{l}\text { Nonrandomized cohort } \\
(n=99)\end{array}$} & \multicolumn{2}{|c|}{ Total $(N=371)$} \\
\hline & $n$ & Mean \pm SD & $n$ & Mean \pm SD & $n$ & Mean \pm SD \\
\hline \multicolumn{7}{|l|}{ Baseline } \\
\hline Days anti-HIV medication taken (past 30 days), $\%$ & 255 & $88.2 \pm 21.6$ & 93 & $93.2 \pm 11.3$ & 348 & $89.5 \pm 19.5$ \\
\hline Doses taken within $2 \mathrm{~h}$ of target time, $\%$ & 254 & $83.9 \pm 24.0$ & 93 & $84.7 \pm 20.5$ & 347 & $84.1 \pm 23.1$ \\
\hline \multicolumn{7}{|l|}{ Week 24} \\
\hline Days anti-HIV medication taken (past 30 days), $\%$ & 225 & $95.5 \pm 8.9$ & 84 & $94.8 \pm 10.2$ & 309 & $95.3 \pm 9.3$ \\
\hline Doses taken within $2 \mathrm{~h}$ of target time, $\%$ & 225 & $90.5 \pm 15.7$ & 84 & $88.5 \pm 15.0$ & 309 & $89.9 \pm 15.5$ \\
\hline
\end{tabular}

$I T T-E$ intention-to-treat-exposed, $S D$ standard deviation 
Table 4 Results by cohort for EQ-5D-3L and EQ-VAS

\begin{tabular}{|c|c|c|c|c|c|c|}
\hline \multirow[t]{2}{*}{ Parameter } & \multicolumn{2}{|c|}{$\begin{array}{l}\text { Randomized cohort }{ }^{\mathrm{a}} \\
(n=272)\end{array}$} & \multicolumn{2}{|c|}{$\begin{array}{l}\text { Nonrandomized cohort } \\
(n=99)\end{array}$} & \multicolumn{2}{|c|}{ Total $(N=371)$} \\
\hline & $n$ & Mean \pm SD & $n$ & Mean \pm SD & $n$ & Mean \pm SD \\
\hline \multicolumn{7}{|l|}{ EQ-VAS } \\
\hline Baseline & 263 & $74.7 \pm 21.6$ & 97 & $70.2 \pm 22.5$ & 360 & $73.5 \pm 21.9$ \\
\hline Week 48 & 222 & $84.4 \pm 15.9$ & 82 & $77.0 \pm 19.8$ & 304 & $82.4 \pm 17.4$ \\
\hline Change from baseline & 218 & $9.8 \pm 21.0$ & 80 & $4.9 \pm 19.5$ & 298 & $8.5 \pm 20.7$ \\
\hline $95 \% \mathrm{CI}^{\mathrm{b}}$ & & $7.0-12.6$ & & $0.6-9.2$ & & $6.1-10.9$ \\
\hline \multicolumn{7}{|l|}{ US-valued utility score } \\
\hline Baseline & 263 & $0.83 \pm 0.17$ & 97 & $0.80 \pm 0.20$ & 360 & $0.82 \pm 0.18$ \\
\hline Week 48 & 223 & $0.85 \pm 0.16$ & 82 & $0.80 \pm 0.20$ & 305 & $0.84 \pm 0.18$ \\
\hline Change from baseline & 218 & $0.02 \pm 0.15$ & 80 & $-0.01 \pm 0.22$ & 298 & $0.01 \pm 0.17$ \\
\hline $95 \% \mathrm{CI}^{\mathrm{b}}$ & & -0.003 to 0.037 & & -0.053 to 0.043 & & -0.008 to 0.030 \\
\hline \multicolumn{7}{|c|}{ UK-referenced utility score } \\
\hline Baseline & 263 & $0.78 \pm 0.24$ & 97 & $0.74 \pm 0.28$ & 360 & $0.77 \pm 0.25$ \\
\hline Week 48 & 223 & $0.81 \pm 0.24$ & 82 & $0.74 \pm 0.29$ & 305 & $0.79 \pm 0.25$ \\
\hline Change from baseline & 218 & $0.03 \pm 0.22$ & 80 & $-0.01 \pm 0.32$ & 298 & $0.02 \pm 0.25$ \\
\hline $95 \% \mathrm{CI}^{\mathrm{b}}$ & & -0.004 to 0.055 & & -0.081 to 0.060 & & -0.012 to 0.044 \\
\hline
\end{tabular}

$B L$ baseline, $C I$ confidence interval, $S D$ standard deviation, $V A S$ visual analog scale

${ }^{a}$ Includes participants who received placebo during the 8-day blinded period

${ }^{\mathrm{b}}$ Post hoc analyses were conducted to determine $95 \%$ CIs
NRC 70.2 \pm 22.5 ; Table 4). At 24 weeks, changes from baseline in the EQ-5D-3L US and UK mean \pm SD utility scores in the RC were $0.02 \pm 0.16$ and $0.02 \pm 0.23$, respectively, and at 48 weeks were $0.02 \pm 0.15$ and $0.03 \pm 0.22$, respectively. In the NRC, changes from baseline in the EQ$5 \mathrm{D}-3 \mathrm{~L}$ US and UK scores at week 24 were $0.03 \pm 0.22$ and $0.04 \pm 0.31$, respectively, and at week 48 were $-0.01 \pm 0.22$ and $-0.01 \pm 0.32$, respectively. Changes from baseline in the mean EQ-VAS scores in the RC and NRC at week 24 were $8.7 \pm 19.5$ and $5.6 \pm 19.3$, respectively, and at week 48 were $9.8 \pm 21.0$ and $4.9 \pm 19.5$, respectively. Across cohorts, 300 of $316(95 \%)$ participants who completed the week 48 visit completed the EQ-5D-3L survey.

\subsection{Functional Assessment of HIV Infection}

The RC had a substantially higher mean total FAHI score at baseline (122.7) than the NRC (114.3), with higher scores across all the subscales (Table 5). In the RC at week 24, positive changes from baseline were observed in the FAHI total score $(6.9$; 95\% CI 4.2-9.7) and the physical well-being (2.7; 95\% CI 1.9-3.6) and emotional well-being subscales (3.2; 95\% CI 2.2-4.2), with little or no change in the function/global well-being, social well-being, and cognitive function subscale scores. In the NRC at week 24, an overall positive change from baseline was observed in the FAHI total score $(2.0 ; 95 \% \mathrm{CI}-3.1-7.2)$ and physical well-being (1.2; 95\% CI - 0.6-3.0) and emotional well-being subscales (1.6; 95\% CI - 0.1-3.3), with little (or negative) change in the function/global well-being, social well-being, and cognitive function subscale scores. Similar changes in FAHI scores were observed at week 48 in the RC and NRC, with positive changes from baseline in total score (RC 5.8; 95\% CI 2.7-9.0; NRC 1.6; 95\% CI - 3.5-6.7) and the physical well-being (RC 2.4; 95\% CI 1.3-3.4; NRC 1.1; 95\% CI - 0.6-2.9) and emotional well-being subscales (RC 2.6; 95\% CI 1.6-3.7; NRC 2.2; 95\% CI 0.3-4.1), with little or no change in the other subscale scores. Across cohorts, 301 of $316(95 \%)$ participants who completed the week 48 visit completed the FAHI survey.

\subsection{Subgroup Analyses}

In general, subgroup analyses demonstrated that participants with lower baseline CD4 + T-cell counts had greater improvements in EQ-5D-3L, EQ-VAS, FAHI total, and FAHI physical and emotional well-being subscale scores at week 48 than participants with higher baseline CD4 + T-cell counts (Tables 6 and 7). In addition, participants with a higher viral load at baseline (HIV-1 RNA $>100,000$ copies/ $\mathrm{mL}$ ) also had greater improvements in EQ-5D-3L, EQ-VAS, FAHI total, and physical and emotional subscale scores at week 48 than participants with lower viral loads at baseline. Generally, participants with fewer years of treatment history at baseline also had greater improvements in EQ-VAS, FAHI total, and physical, emotional, functional, and cognitive subscales. 
Table 5 Results by cohort for Functional Assessment of HIV Infection instrument

\begin{tabular}{|c|c|c|c|c|c|c|}
\hline \multirow[t]{2}{*}{ Parameter } & \multicolumn{2}{|c|}{$\begin{array}{l}\text { Randomized cohort }{ }^{\mathrm{a}} \\
(n=272)\end{array}$} & \multicolumn{2}{|c|}{$\begin{array}{l}\text { Nonrandomized cohort } \\
(n=99)\end{array}$} & \multicolumn{2}{|c|}{ Total $(N=371)$} \\
\hline & $n$ & Mean \pm SD & $n$ & Mean \pm SD & $n$ & Mean \pm SD \\
\hline \multicolumn{7}{|l|}{ Total score } \\
\hline Baseline & 261 & $122.7 \pm 28.6$ & 97 & $114.3 \pm 34.1$ & 358 & $120.4 \pm 30.4$ \\
\hline Week 48 & 223 & $128.7 \pm 28.9$ & 81 & $117.6 \pm 32.8$ & 304 & $125.8 \pm 30.3$ \\
\hline Change from baseline & 216 & $5.8 \pm 23.5$ & 79 & $1.6 \pm 22.7$ & 295 & $4.7 \pm 23.4$ \\
\hline $95 \% \mathrm{CI}^{\mathrm{b}}$ & & $2.7-9.0$ & & -3.5 to 6.7 & & $2.0-7.4$ \\
\hline \multicolumn{7}{|l|}{ Physical well-being } \\
\hline Baseline & 263 & $30.7 \pm 8.6$ & 97 & $29.0 \pm 9.9$ & 360 & $30.2 \pm 8.9$ \\
\hline Week 48 & 225 & $33.2 \pm 7.2$ & 82 & $31.1 \pm 8.3$ & 307 & $32.6 \pm 7.6$ \\
\hline Change from baseline & 220 & $2.4 \pm 7.7$ & 80 & $1.1 \pm 8.0$ & 300 & $2.0 \pm 7.8$ \\
\hline $95 \% \mathrm{CI}^{\mathrm{b}}$ & & $1.3-3.4$ & & -0.6 to 2.9 & & $1.2-2.9$ \\
\hline \multicolumn{7}{|l|}{ Emotional well-being } \\
\hline Baseline & 263 & $26.3 \pm 9.3$ & 97 & $24.9 \pm 10.1$ & 360 & $25.9 \pm 9.5$ \\
\hline Week 48 & 225 & $28.8 \pm 9.1$ & 81 & $27.5 \pm 9.3$ & 306 & $28.5 \pm 9.1$ \\
\hline Change from baseline & 220 & $2.6 \pm 8.1$ & 79 & $2.2 \pm 8.5$ & 299 & $2.5 \pm 8.2$ \\
\hline $95 \% \mathrm{CI}^{\mathrm{b}}$ & & $1.6-3.7$ & & $0.3-4.1$ & & $1.6-3.4$ \\
\hline \multicolumn{7}{|c|}{ Function and global well-being } \\
\hline Baseline & 263 & $35.3 \pm 9.9$ & 97 & $31.8 \pm 12.1$ & 360 & $34.4 \pm 10.7$ \\
\hline Week 48 & 225 & $36.4 \pm 10.4$ & 82 & $31.7 \pm 12.0$ & 307 & $35.2 \pm 11.1$ \\
\hline Change from baseline & 220 & $1.3 \pm 10.4$ & 80 & $-0.9 \pm 9.6$ & 300 & $0.7 \pm 10.2$ \\
\hline $95 \% \mathrm{CI}^{\mathrm{b}}$ & & -0.1 to 2.6 & & -3.0 to 1.3 & & -0.5 to 1.8 \\
\hline \multicolumn{7}{|l|}{ Social well-being } \\
\hline Baseline & 263 & $22.1 \pm 7.5$ & 97 & $20.6 \pm 8.5$ & 360 & $21.7 \pm 7.8$ \\
\hline Week 48 & 224 & $21.9 \pm 8.0$ & 82 & $19.5 \pm 9.6$ & 306 & $21.3 \pm 8.5$ \\
\hline Change from baseline & 219 & $0.0 \pm 6.4$ & 80 & $-0.9 \pm 6.5$ & 299 & $-0.3 \pm 6.4$ \\
\hline $95 \% \mathrm{CI}^{\mathrm{b}}$ & & -0.9 to 0.8 & & -2.4 to 0.5 & & -1.0 to 0.4 \\
\hline \multicolumn{7}{|l|}{ Cognitive functioning } \\
\hline Baseline & 261 & $8.4 \pm 2.7$ & 97 & $7.9 \pm 3.0$ & 358 & $8.3 \pm 2.8$ \\
\hline Week 48 & 224 & $8.4 \pm 2.7$ & 82 & $8.2 \pm 2.6$ & 306 & $8.4 \pm 2.7$ \\
\hline Change from baseline & 217 & $0.0 \pm 2.8$ & 80 & $0.3 \pm 2.7$ & 297 & $0.1 \pm 2.8$ \\
\hline $95 \% \mathrm{CI}^{\mathrm{b}}$ & & -0.4 to 0.4 & & -0.3 to 0.9 & & -0.2 to 0.4 \\
\hline
\end{tabular}

$C I$ confidence interval, $S D$ standard deviation

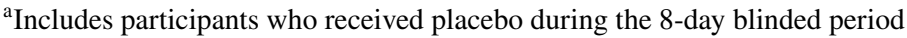

${ }^{\mathrm{b}}$ Post hoc analyses were conducted to determine $95 \% \mathrm{CI}$

\section{Discussion}

The addition of the HIV-1 attachment inhibitor, fostemsavir, and optimization of background regimens resulted in improvements in HRQOL PROs in HTE PLWH. Both the $\mathrm{RC}$ and the NRC had advanced disease, including those with low baseline CD4 + T-cell counts and a high proportion of participants with a history of AIDS, which was reflected in low HRQOL baseline scores. Relative to the RC, a greater proportion of participants in the NRC had CD4+ T-cell counts $<20$ cells $/ \mathrm{mm}^{3}$, which may have contributed to their lower HRQOL scores at baseline.

Despite $<100 \%$ adherence as assessed by M-MASRI, rates of virologic suppression were maintained between week 24 and week 48. Few changes were observed from baseline in the EQ-5D-3L assessment at weeks 24 and 48. However, improvements in scores from baseline in the EQ-VAS and FAHI assessments at weeks 24 and 48 were observed in both cohorts. The FAHI is an HIV-specific measure that reports on specific components (e.g., physical, emotional, and social well-being) and has been shown to be a useful tool, in addition to the traditional clinical endpoints, to evaluate HIV-1 treatments [10]. Through week 48 , FAHI showed improvements from baseline in total score and physical well-being and emotional well-being subscale scores, with little or no change in function/global well-being, social well-being, and cognitive function subscale scores. These FAHI subscale results are consistent with results in 


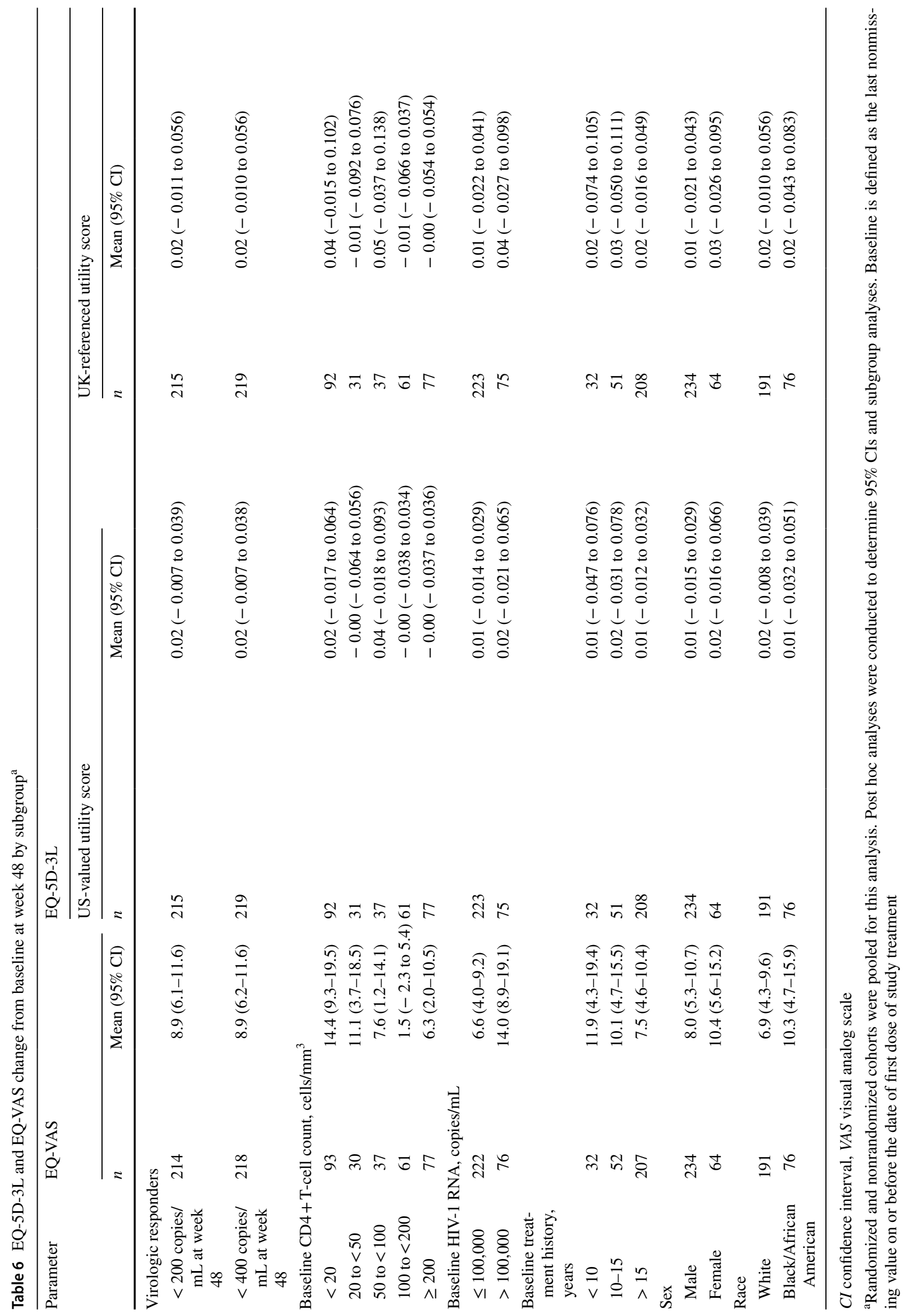




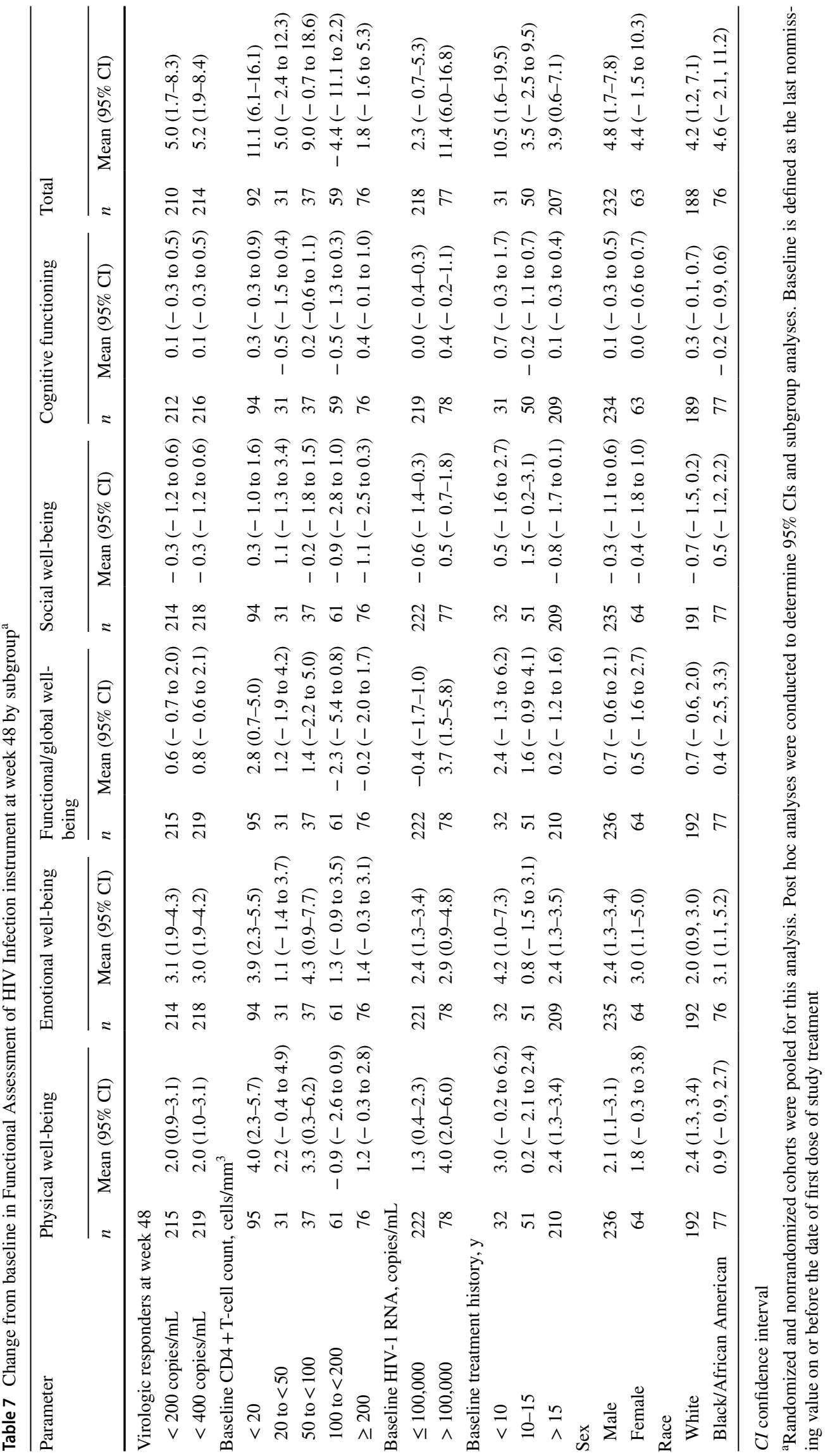


the DUET and POWER clinical trials, which had comparable study populations $[10,15,25,26]$. The DUET trial assessed the efficacy and safety of etravirine plus OBT in participants with multidrug-resistant HIV-1 infection [15, 27, 28], whereas the POWER 1 and 2 trials evaluated the efficacy and safety of darunavir-ritonavir plus OBT in treatment-experienced PLWH [25]. The FAHI data from these studies showed increases from baseline at 24 or 48 weeks in total FAHI score and physical well-being and function and global well-being subscale scores, with little or no change in the other subscales for participants treated with etravirine in the DUET trial and participants treated with darunavir-ritonavir in the POWER trials [16, 26, 29]. In addition, in the DUET trial, virologic responders had clinically significant improvements in total FAHI score and physical well-being and emotional well-being subscale scores [10].

Fostemsavir is indicated for HTE PLWH, who are often immunosuppressed. The PRO data from the BRIGHTE study demonstrated that participants treated with fostemsavir experienced improvements in HRQOL, with greater improvements observed in those with higher viral loads and lower CD4 + T-cell counts, i.e., individuals who were the most immunocompromised at baseline.

One limitation of this study was the variation in OBT taken with fostemsavir, which limits the interpretability of fostemsavir in combination with specific antiretroviral background therapies. In addition, although improvements in PROs from baseline were observed with the addition of fostemsavir, these outcomes were not compared with a placebo or other comparator group because, after day 8 , all participants received fostemsavir and OBT. Furthermore, this study was not specifically designed to demonstrate change in PROs from baseline through week 48 or differences in PROs between subgroups. Clinically important difference values for HRQOL assessments have previously been derived using anchor- and distribution-based methods; future work could look to estimate similar thresholds in the HTE population [30]. Although patient-reported dosing diaries recorded treatment adherence through 48 weeks, the M-MASRI questionnaire only assessed treatment adherence at week 24 . Of note, EQ-5D-3L US- and UK-referenced utility scores were derived from US and UK value sets and applied across all data despite differences in value sets across countries. Finally, limitations of the EQ-5D-3L include concern over discriminating small-to-moderate differences in health status and ceiling effects [23].

These results from the BRIGHTE study demonstrated improvements in HRQOL in a diverse population of HTE PLWH who often have extensive ART drug resistance and limited treatment options. As most of the HTE population has experienced years of ART and disease progression while aging, HRQOL is a particularly important measure for public health as this population continues to grow. Symptomatic disease and decreased CD4+ T-cell counts have been associated with lower HRQOL scores [13, 31]. Furthermore, data from the multinational OPTIMA trial showed that serially treated PLWH with advanced disease experienced high rates of non-AIDS-related serious AEs (60.96 per 100 patientyears), leading to immediate and significant detriments to HRQOL [32]. Improvements in HRQOL may increase adherence to treatment regimens, thereby helping to lead to achievements in long-term treatment success. The ongoing BRIGHTE study will allow for longer-term follow-up that can enhance our understanding of PROs in PLWH receiving ART. These HRQOL data, in addition to the efficacy and safety results of the BRIGHTE study, support the use of fostemsavir in HTE individuals living with multidrugresistant HIV-1 infection who presently have few remaining therapeutic options.

Acknowledgements The authors acknowledge Kathy Zhai (GlaxoSmithKline) for her contributions to the study. Editorial assistance was provided under direction of the authors by MedThink SciCom and was funded by ViiV Healthcare.

\section{Declarations}

Funding This study was funded by ViiV Healthcare.

Conflicts of interest S-JA and MW are employees of and own stock in GlaxoSmithKline. MM was an employee of ViiV Healthcare when the work was conducted, is now with Health Analytics and Outcomes, London, UK, and owns/owned stock in GlaxoSmithKline. DC is president of FACIT.org, which licenses FAHI, and has been a consultant to ViiV Healthcare. RG has received research support from ViiV Healthcare, Amgen, and Gilead Sciences; consulting fees from Thera Technologies as member of an advisory board; and speaker fees from ViiV Healthcare. DH has received speaker fees and consulting fees from ViiV Healthcare as a member of an advisory board. WT is an employee of Southern California Permanente Medical Group and has received institutional grants from ViiV Healthcare, Bristol-Myers Squibb, Gilead, Dynavax, and Merck. AC, AP, CL, PA, and ML are employees of ViiV Healthcare and own stock in GlaxoSmithKline.

Ethics approval The study was performed in accordance with Good Clinical Practice, as defined in the International Conference on Harmonisation and in accordance with the ethical principles underlying European Union Directive 2001/20/EC and the United States Code of Federal Regulations, Title 21, Part 50. Each site conducted the protocol with oversight from an institutional review board or independent ethics committee.

Consent to participate All participants provided informed consent in agreement with the Declaration of Helsinki principles.

Consent for publication Not applicable.

Availability of data and material Anonymized individual participant data and study documents can be requested for further research from http://www.clinicalstudydatarequest.com.

Code availability Not applicable. 
Authors' contributions CL contributed to the conception of the study. CL contributed to the design of the study. RG, DH, WT, AP, CL, and PA contributed to the acquisition of data. SJA, MM, MW, AC, AP, $\mathrm{CL}$, and PA contributed to the analysis of data. SJA, MM, DC, AC, AP, CL, and PA contributed to the interpretation of data. SJA and MM contributed to the drafting of the manuscript. All listed authors meet the criteria for authorship set forth by the International Committee of Medical Journal Editors. All authors contributed to critically revising the manuscript for important intellectual content and approve the manuscript for publication.

Open Access This article is licensed under a Creative Commons Attribution-NonCommercial 4.0 International License, which permits any non-commercial use, sharing, adaptation, distribution and reproduction in any medium or format, as long as you give appropriate credit to the original author(s) and the source, provide a link to the Creative Commons licence, and indicate if changes were made. The images or other third party material in this article are included in the article's Creative Commons licence, unless indicated otherwise in a credit line to the material. If material is not included in the article's Creative Commons licence and your intended use is not permitted by statutory regulation or exceeds the permitted use, you will need to obtain permission directly from the copyright holder. To view a copy of this licence, visit http://creativecommons.org/licenses/by-nc/4.0/.

\section{References}

1. Palella FJ Jr, Delaney KM, Moorman AC, et al, and the HIV Outpatient Study Investigators. Declining morbidity and mortality among patients with advanced human immunodeficiency virus infection. N Engl J Med. 1998;338(13):853-60.

2. Brown J, Chien C, Timmins P, et al. Compartmental absorption modeling and site of absorption studies to determine feasibility of an extended-release formulation of an HIV-1 attachment inhibitor phosphate ester prodrug. J Pharm Sci. 2013;102(6):1742-51.

3. Langley DR, Kimura SR, Sivaprakasam P, et al. Homology models of the HIV-1 attachment inhibitor BMS-626529 bound to gp120 suggest a unique mechanism of action. Proteins. 2015;83(2):331-50.

4. Ray N, Hwang C, Healy MD, et al. Prediction of virological response and assessment of resistance emergence to the HIV-1 attachment inhibitor BMS-626529 during 8-day monotherapy with its prodrug BMS-663068. J Acquir Immune Defic Syndr. 2013;64(1):7-15.

5. Nowicka-Sans B, Gong Y-F, McAuliffe B, et al. In vitro antiviral characteristics of HIV-1 attachment inhibitor BMS-626529, the active component of the prodrug BMS-663068. Antimicrob Agents Chemother. 2012;56(7):3498-507.

6. Li Z, Zhou N, Sun Y, et al. Activity of the HIV-1 attachment inhibitor BMS-626529, the active component of the prodrug BMS663068, against CD4-independent viruses and HIV-1 envelopes resistant to other entry inhibitors. Antimicrob Agents Chemother. 2013;57(9):4172-80.

7. Rukobia [prescribing information]. Research Triangle Park, NC: ViiV Healthcare; 2020.

8. Kozal M, Aberg J, Pialoux G, et al. Fostemsavir in adults with multidrug-resistant HIV-1 infection. N Engl J Med. 2020;382(13):1232-43.

9. Powers JH III, Howard K, Saretsky T, et al. Patient-reported outcome assessments as endpoints in studies in infectious diseases. Clin Infect Dis. 2016;63(suppl 2):S52-6.
10. Viala-Danten M, Dubois D, Gilet H, Martin S, Peeters K, Cella D. Psychometric evaluation of the functional assessment of HIV Infection (FAHI) questionnaire and its usefulness in clinical trials. Qual Life Res. 2010;19(8):1215-27.

11. Nachega JB, Marconi VC, van Zyl GU, et al. HIV treatment adherence, drug resistance, virologic failure: evolving concepts. Infect Disord Drug Targets. 2011;11(2):167-74.

12. Struble K, Murray J, Cheng B, Gegeny T, Miller V, Gulick R. Antiretroviral therapies for treatment-experienced patients: current status and research challenges. AIDS. 2005;19(8):747-56.

13. Campsmith ML, Nakashima AK, Davidson AJ. Self-reported health-related quality of life in persons with HIV infection: results from a multi-site interview project. Health Qual Life Outcomes. 2003;1:12.

14. Bucciardini R, Pugliese K, Weimer L, et al. Relationship between health-related quality of life measures and high HIV viral load in HIV-infected triple-class-experienced patients. HIV Clin Trials. 2014;15(4):176-83.

15. Cella D, Gilet H, Viala-Danten M, Peeters K, Dubois D, Martin S. Effects of etravirine versus placebo on health-related quality of life in treatment-experienced HIV patients as measured by the functional assessment of human immunodeficiency virus infection (FAHI) questionnaire in the DUET trials. HIV Clin Trials. 2010;11(1):18-27.

16. Cella DF, McCain NL, Peterman AH, Mo F, Wolen D. Development and validation of the Functional Assessment of Human Immunodeficiency Virus Infection (FAHI) quality of life instrument. Qual Life Res. 1996;5(4):450-63.

17. Boyd MA, Hill AM. Clinical management of treatment-experienced, HIV/AIDS patients in the combination antiretroviral therapy era. Pharmacoeconomics. 2010;28(suppl 1):17-34.

18. Burgoyne RW, Tan DHS. Prolongation and quality of life for HIV-infected adults treated with highly active antiretroviral therapy (HAART): a balancing act. J Antimicrob Chemother. 2008;61(3):469-73.

19. Walsh JC, Mandalia S, Gazzard BG. Responses to a 1 month self-report on adherence to antiretroviral therapy are consistent with electronic data and virological treatment outcome. AIDS. 2002;16(2):269-77.

20. EuroQol. EQ-5D-3L user guide. EuroQol Research Foundation. 2015. https://euroqol.org/publications/user-guides/. Accessed 15 Apr 2019.

21. Miners A, Phillips A, Kreif N, et al. Health-related quality-of-life of people with HIV in the era of combination antiretroviral treatment: a cross-sectional comparison with the general population. Lancet HIV. 2014;1(1):e32-40.

22. Byrne S, Petry NM. Reliability and validity of the Functional Assessment of Human Immunodeficiency Virus Infection (FAHI) in patients with drug and alcohol use disorders. AIDS Care. 2013;25(1):118-25.

23. Janssen MF, Pickard AS, Golicki D, et al. Measurement properties of the EQ-5D-5L compared to the EQ-5D-3L across eight patient groups: a multi-country study. Qual Life Res. 2013;22(7):1717-27.

24. Nan L, Johnson JA, Shaw JW, Coons SJ. A comparison of EQ-5D index scores derived from the US and UK population-based scoring functions. Med Decis Making. 2007;27(3):321-6.

25. Clotet B, Bellos N, Molina J-M, et al. Efficacy and safety of darunavir-ritonavir at week 48 in treatment-experienced patients with HIV-1 infection in POWER 1 and 2: a pooled subgroup analysis of data from two randomised trials. Lancet. 2007;369(9568):1169-78.

26. Dubois D, Smets E, Vangeneugden T, et al. Improved quality of life in treatment-experienced HIV patients treated with TMC114/r vs control protease inhibitors: results of POWER 1 and 2 functional assessment of HIV infection (FAHI). Poster presented 
at: 16th International AIDS Conference; August 13-18, 2006; Toronto, Canada.

27. Madruga JV, Cahn P, Grinsztejn B, et al. Efficacy and safety of TMC125 (etravirine) in treatment-experienced HIV-1-infected patients in DUET-1: 24-week results from a randomised, doubleblind, placebo-controlled trial. Lancet. 2007;370(9581):29-38.

28. Lazzarin A, Campbell T, Clotet B, et al. Efficacy and safety of TMC125 (etravirine) in treatment-experienced HIV-1-infected patients in DUET-2: 24-week results from a randomised, doubleblind, placebo-controlled trial. Lancet. 2007;370(9581):39-48.

29. Gakhar H, Kamali A, Holodniy M. Health-related quality of life assessment after antiretroviral therapy: a review of the literature. Drugs. 2013;73(7):651-72.
30. Wyrwich KW, Bullinger M, Aaronson N, Hays RD, Patrick DL, Symonds T. Estimating clinically significant differences in quality of life outcomes. Qual Life Res. 2005;14(2):285-95.

31. Briongos Figuero LS, Bachiller Luque P, Palacios Martín T, González Sagrado M, Eiros Bouza JM. Assessment of factors influencing health-related quality of life in HIV-infected patients. HIV Med. 2011;12(1):22-30.

32. Anis AH, Nosyk B, Sun H, et al. Quality of life of patients with advanced HIV/AIDS: measuring the impact of both AIDSdefining events and non-AIDS serious adverse events. J Acquir Immune Defic Syndr. 2009;51(5):631-9. 